\title{
The ALARA concept in pediatric oncology
}

\author{
Stephan D. Voss • Gregory H. Reaman • Sue C. Kaste • \\ Thomas L. Slovis
}

Received: 21 August 2009 / Accepted: 26 August 2009 /Published online: 30 September 2009

(C) Springer-Verlag 2009

For the past several years, the Society for Pediatric Radiology (SPR) has successfully conducted ALARA conferences emphasizing the importance of radiation safety in pediatric imaging, providing a valuable forum for pediatric radiologists, practicing general radiologists, and interested clinicians to review specific concepts relevant to the ALARA (as low as reasonably achievable) principle [1-4]. In 2008 the focus of the ALARA symposium was pediatric oncology imaging. Imaging endpoints and sophisticated imaging studies are increasingly being incorporated into oncologic treatment protocols and clinical trials. This symposium underscored the need for increased awareness of the potential for radiation-related injury from repeated exposure to ionizing radiation. Such a forum brought together key members of the pediatric imaging and pediatric oncology communities and highlighted the need for

S. D. Voss $(\bowtie)$

Department of Radiology, Children's Hospital Boston,

Harvard Medical School,

300 Longwood Ave.,

Boston, MA 02115, USA

e-mail: Stephan.Voss@childrens.harvard.edu

\section{G. H. Reaman}

Department of Pediatrics, The George Washington University

School of Medicine and Health Sciences,

Children's Oncology Group,

Washington, DC, USA

\section{S. C. Kaste}

Radiological Sciences,

St. Jude Children's Research Hospital MSN \#220,

Memphis, TN, USA

\section{T. L. Slovis}

Departments of Radiology and Pediatrics, Wayne State University

School of Medicine, Children's Hospital of Michigan,

Detroit, MI, USA evidence-based methods to guide diagnostic and surveillance imaging and to identify opportunities for interdisciplinary investigation.

Numerous reports have emphasized that even low doses of radiation exposure, such as those occurring during radiological examinations, can lead to long-term adverse health outcomes, most notably increases in risk of malignancy $[5,6]$. The Society for Pediatric Radiology has successfully launched the Image Gently campaign, highlighting the importance of radiation safety in pediatric imaging [7]. What began largely as an initiative within the Society for Pediatric Radiology has expanded well beyond our own society and now has representation from a large number of imaging organizations, as well as representation from major manufacturers [8]. Although the ALARA concept has been widely embraced by the pediatric radiology imaging community, it is essential that this awareness be expanded outside the imaging community to our referring clinicians. Pediatric oncology patients are some of the most aggressively treated and intensively imaged patients in pediatric practice. While imaging is central to the diagnosis, treatment and management of these critically ill children, the large number of serial-and frequently high-dose - examinations has the potential to contribute to considerable cumulative radiation exposure.

Two recent studies have provided direct evidence to support this concern, demonstrating significant cumulative effective doses in multiple tumor subgroups, with neuroblastoma and lymphoma patients receiving the largest radiation burden $[9,10]$. These observations were attributable to the large number of nuclear medicine and CT examinations that oncology patients routinely undergo. Because these patients are concurrently receiving cytotoxic chemotherapy and frequently radiation therapy, it may be difficult to calculate an excess relative risk attributable to 
the additional radiation exposure from diagnostic imaging procedures. Nonetheless, all available data indicate that these increases in cumulative radiation exposure may be significant. As this ALARA symposium emphasized, weighing these risks against the potential benefits of an imaging study requires thoughtful collaboration between pediatric radiologists and pediatric oncologists [11]. It is through such teamwork that the most appropriate imaging studies can be integrated into oncology treatment protocols, with the timing and frequency of imaging being optimized to best accomplish treatment and disease-specific response assessments.

\section{Strategies for dose reduction}

A number of strategies can be effectively implemented in order to perform necessary studies while still adhering to the ALARA principle.

\section{Technical considerations}

Technical factors are easily controlled and should be a focus for dose reduction in any radiology practice. CT scanners are readily available in nearly all major medical centers and are generally free of technical and operatordependent variability. There are published guidelines - now readily accessible on ImageGently.org-for reducing CT dose based on age and/or weight [12, 13]. Some CT manufacturers provide automatic $\mathrm{mA}$ adjustment algorithms with their scanners, thereby tailoring the effective radiation exposure to the attenuation characteristics of specific tissues. Members of the SPR Image Gently taskforce are working with manufacturers to develop better estimates for calculating pediatric patient radiation dose, based upon pediatric anthropomorphic phantom models that better reflect the various sizes, shapes and attenuation properties of children's bodies [8].

It should also be standard practice to eliminate unnecessary imaging sequences that are not contributing significantly to diagnostic, treatment, or clinical management decisions or to endpoints in clinical trials. For example, unenhanced CT scanning followed by contrast-enhanced scanning of the abdomen/pelvis plays little role in the diagnosis and staging of oncology patients and virtually doubles the radiation exposure. In addition, there are few indications that justify routine multi-phasic contrastenhanced CT scanning in pediatrics. This technique should be reserved for complex surgical planning, and can often be replaced by a properly timed single-phase CT angiogram.

Perhaps the most important technical factor to consider is whether alternative imaging modalities (e.g., US, MRI) can provide comparable diagnostic information while obviating exposure to ionizing radiation [14]. As an example of how alternative imaging strategies can be implemented in pediatric oncologic imaging consider the routine staging of patients with suspected lymphoma in the U.S. as compared to Europe. Patients in the United States are typically staged and subsequently monitored using contrast-enhanced CT of the neck, chest, abdomen and pelvis. In many European countries, imaging of the abdomen has been largely replaced by MRI, with CT being reserved primarily for assessment of the lung parenchyma and mediastinum [15]. With advances in rapid MRI techniques, we could nearly completely replace CT with MRI in evaluating these patients. Similarly, imaging of the primary tumor in Wilms tumor patients, both at diagnosis and following therapy, can be effectively accomplished with US and MRI. The MRI signal characteristics of the tumor may also help to distinguish malignant disease from persistent nephrogenic rests [16]. In patients with neuroblastoma, MRI-particularly with wholebody imaging techniques - can provide valuable information about the primary tumor and potential sites of marrow involvement [17].

Although the ALARA oncology symposium did not specifically focus on the various minimally invasive imaging techniques used in the care of children with cancer, it is well established that imaging technologies can effectively guide diagnosis and management, even in the research setting, in pediatric oncology patients, oftentimes eliminating the need for higher-risk surgical procedures. Regardless of the method chosen, it is incumbent upon the interventional radiologist, oncologist, and surgeon to collaboratively develop the most effective approach to obtaining diagnostic material [18].

Developing a rational basis for staging and post-treatment response assessment

As highlighted by the presenters at the recent ALARA Oncology conference, developing an evidence-based approach to imaging pediatric oncology patients is of paramount importance. The successes in treating childhood cancer during the last several decades have been accomplished primarily through multi-center and multidisciplinary clinical and basic research. The development of new treatment modalities has been systematic, rigorous, and based on sound preclinical and clinical evidence [19]. Despite this well-established approach to developing new therapies, similar rigor has not been universally applied to the development of response assessments. With newer targeted and molecular therapeutic approaches rapidly entering the clinical research arena, prospective development of improved and specific diagnostic imaging techniques is necessary to provide the response assessments needed for risk-adjusted treatment regimens [20]. 
Determining the most appropriate imaging techniques for post-treatment response assessment is the subject of much debate. The Response Evaluation Criteria in Solid Tumors (RECIST) are used in most adult protocols and in many pediatric protocols. As has been noted by many investigators, the most common non-CNS pediatric solid tumors (neuroblastoma, Wilms tumor, and lymphoma) are not well-suited to RECIST measurement criteria [21, 22]. Pediatric bone tumors, including Ewing sarcoma and osteosarcoma, are not readily measurable by traditional RECIST criteria and there is no generally acceptable standard for measuring bone tumor size. These inconsistencies are compounded when soft-tissue and bone involvement co-exist and when the margins of peritumoral edema preclude accurate measurements of tumor size. In such settings, the introduction of functional imaging techniques such as dynamic enhanced MRI, diffusion-weighted MRI, and FDG-PET scanning to direct response assessment may prompt replacement of current anatomic measures of disease assessment such as RECIST. These efforts, however, cannot be organized and implemented exclusively by radiologists, but will require a close collaboration between radiology and oncology investigators.

The use of FDG-PET imaging for staging and assessing response to therapy has gained increasing acceptance among pediatric oncologists. In Hodgkin lymphoma, accumulating evidence suggests that elimination of tumor metabolic activity, as determined by FDG-PET, may be the most important predictor of event-free and overall survival $[15,23-26]$. The use of either CT or MRI in conjunction with these PET scans improves the specificity of a judiciously timed PET evaluation and provides essential anatomic correlation needed to guide subsequent treatment decisions, such as radiation treatment planning [15]. It is now common practice for most pediatric oncology patients to receive a PET/CT scan during the initial staging evaluation. Many of these children will also undergo a diagnostic contrast-enhanced $\mathrm{CT}$ for their initial diagnostic workup. Obtaining a low-dose CT scan for the purposes of attenuation correction of the PET imaging data is necessary; however, obtaining a repeat diagnostic contrast-enhanced $\mathrm{CT}$ at the time of PET imaging may not justifiable.

Another example of increased radiation exposure from imaging, without clear diagnostic or therapeutic benefit, is the practice of performing ${ }^{99 \mathrm{~m}} \mathrm{Tc}-\mathrm{MBP}$ bone scans in patients with suspected neuroblastoma and/or lymphoma. Several studies suggest that neuroblastoma staging and response assessment may be effectively accomplished with MIBG scintigraphy coupled with whole-body MRI and/or CT [27], although an increasing role for FDG-PET in neuroblastoma has also been reported [28]. There are few instances (with the notable exception of stage IV-S disease) where disease is detected only by bone scintigraphy but is not seen by MIBG and/or CT/MRI [29]. Similarly, in lymphoma there is little evidence that bone scanning provides additional information beyond that already attainable with FDG-PET imaging with respect to staging and response assessment.

Any post-treatment response assessment must be both sensitive and specific. In addition, both functional and anatomic assessments of response and surveillance for disease recurrence will require a better understanding of the natural history of a disease in order to direct most optimally the appropriate use of imaging studies. An example of a rational development of staging and post-treatment response assessment is in the setting of pediatric Wilms tumor. Over the course of multiple clinical trials coordinated initially through the National Wilms Tumor Study Group and subsequently through the Children's Oncology Group, the clinical stage of the patient was determined, in part, by lung lesions visualized on chest radiograph. Lung lesions detected by chest $\mathrm{CT}$ alone did not affect the clinical stage of the patient. Subsequent studies, both in the U.S. and in Europe, revealed that patients with pulmonary metastatic disease evident on $\mathrm{CT}$, independent of whether that disease was detectable by conventional radiography, had a higher incidence of disease progression and lower overall survival [30, 31]. This led to modifications in treatment protocol and the current recommendations for staging chest $\mathrm{CT}$ in patients with Wilms tumor.

\section{Developing a rational approach for surveillance imaging}

With success in treating many childhood cancers, renewed emphasis has been placed on minimizing the late effects of therapy. As an example, Hodgkin lymphoma patients now enjoy greater than $90 \%$ overall survival in most settings. Despite this success, these patients may receive as many as 15 CT scans during a routine course of therapy and posttherapy follow-up. There is little doubt that CT scans obtained during the early acute stages of the patient's treatment are necessary. However, it may be possible to reduce the number of scans being performed in certain groups of patients with low-stage disease, disease that has responded rapidly to therapy, and in patients who have had no evidence of disease recurrence in the early posttreatment period and in whom the likelihood of disease relapse is declining. In addition, it is unclear whether imaging studies alone identify sites of disease relapse that were not identified or suspected clinically. Thus, new strategies should be developed based on contemporary knowledge of the most likely sites and time courses of relapse of the various pediatric malignancies. Again, this will require a close collaboration between pediatric imagers and pediatric oncologists to optimize the imaging strategy for long-term follow-up in an effort to minimize late effects that may result from increased radiation exposure. 


\section{Workforce issues}

Strategies for improving and optimizing the use of imaging in the diagnosis, treatment and long-term monitoring of pediatric oncology patients will necessarily create additional challenges for the pediatric radiology workforce [14]. Most of these children are being treated on multi-center collaborative clinical trials. Imaging studies being obtained as part of these clinical trials rely on reproducible measurements to guide response and subsequent therapeutic decision-making. This creates challenges at the treating institution, where busy radiologists are called upon to make time-consuming measurements and to ensure that imaging studies are performed in accordance with requirements outlined in the treatment protocol. Radiologists designing the imaging protocols that are to be incorporated into multicenter clinical trials are therefore charged with developing standardized protocols that minimize inter-institutional variability and with optimizing these protocols for simplicity and reproducibility.

There is little doubt that complex imaging examinations that require increased scan time and radiologist input will rapidly fall out of favor with busy pediatric radiologists. Complex or experimental imaging techniques should be reserved for specific therapeutic studies and may be limited to certain treatment centers, preferably with inclusion of an imaging co-investigator at the participating study sites in order to ensure the imaging examinations are being performed in accordance with the experimental study design. This has been accomplished with some success, for example, in an ongoing trial in pediatric leukemia patients, where diffusion tensor imaging is being used to assess structural abnormalities in the brain for correlation with neuro-cognitive function in patients receiving neurotoxic chemotherapy and radiation.

An additional challenge facing pediatric imagers is the transfer of images for central review. The Children's Oncology Group has enjoyed a close relationship with the Quality Assurance Review Center (QARC), where imaging studies requiring central review have historically been collected [32]. Members of the clinical trial study committee meet and review imaging and clinical data collected from patients on trials. COG will also be expanding electronic access to the Children's Oncology Data Center, such that each institution will ultimately have the ability to confidentially de-identify and directly transfer images from their PACS system to the imaging data center in a HIPAAcompliant manner. This latter process has been implemented in the 21 institutions that make up the Children's Oncology Group (COG) Phase 1/Pilot Consortium [33], and allows for de-identified images to be collected and reviewed by the study committee at the completion of a clinical trial, or to be directly transferred to the study radiologist for contempora- neous central review when clinical trial decisions mandate rapid central review of imaging.

Although these image transfers can be fairly easily accomplished with nearly all existing PACS systems, there will be a requirement for an initial up-front investment in informatics time and support in order to expand existing COG data networks to include transfer of imaging data. It will be important for radiologists and pediatric oncologists at individual institutions to work together to develop this infrastructure. We have little hope of optimizing and standardizing protocols that can be applied across multiple institutions and across the larger pediatric oncology community if we are not able to transfer images between institutions and to central review sites. Such infrastructure is necessary if radiologists are to play a role in ensuring that study endpoints are being reached.

Working within the Children's Oncology Group, members of the Society for Pediatric Radiology serve on the diagnostic imaging committee, on multiple disease-specific committees, and are key players in facilitating the process of image transfer and central review. With the increased use of relatively rapid/live central review, it will be possible to provide feedback to local sites, guiding real-time treatment decision-making, and to create a mechanism for close collaboration among institutional radiologists, treating clinicians, and committee radiologists.

\section{Conclusion}

In summary, the ALARA Oncology symposium emphasized the importance of developing close collaborations between pediatric oncologists and pediatric radiologists. With well-recognized institutional constraints and limitations on time, space and fiscal resources, developing the infrastructure to accomplish many of these goals will be challenging. Nonetheless, it is our obligation to perform the safest and most appropriate imaging examinations and to extract as much information as possible from the imaging data. Developing a coordinated approach to critically evaluating and implementing diagnostic imaging procedures in pediatric oncology must be a focus of pediatric clinical cancer research and pediatric imaging research.

In beginning to integrate imaging with therapeutic research in childhood cancer the Children's Oncology Group and the Society for Pediatric Radiology confirm that imaging gently, imaging safely and imaging appropriately for all oncology patients will require a close collaboration between the SPR and the COG. The COG, as a working group, is a vehicle through which to apply the best use of imaging resources in response to clinically relevant questions. The SPR, as an imaging society, has an opportunity to serve the pediatric oncology community by 
collaborating actively in the design and conduct of studies, by providing educational resources, training workshops and a common voice to the American College of Radiology, the National Institutes of Health, and the technical manufacturers and pharmaceutical industry. Through the Image Gently initiative, it is our hope, as emphasized by the success of the ALARA oncology symposium, that these goals can be realized and that the concept of Imaging Gently in oncology will serve to strengthen the relationship between pediatric radiologists and pediatric oncologists in their joint efforts to improve the outcome for children with cancer.

\section{References}

1. Slovis TL (2002) The ALARA (as low as reasonably achievable) concept in pediatric CT intelligent dose reduction. Multidisciplinary conference organized by the Society of Pediatric Radiology. Pediatr Radiol 32:217-313

2. Frush DP, Frush KS (2008) The ALARA concept in pediatric imaging: building bridges between radiology and emergency medicine: consensus conference on imaging safety and quality for children in the emergency setting, Feb. 23-24, 2008, Orlando, FL-Executive Summary. Pediatr Radiol 38(Suppl 4):S629-S632

3. Strauss KJ, Kaste SC (2006) The ALARA (as low as reasonably achievable) concept in pediatric interventional and fluoroscopic imaging: striving to keep radiation doses as low as possible during fluoroscopy of pediatric patients - a white paper executive summary. Pediatr Radiol 36(Suppl 2):110-112

4. Willis CE, Slovis TL (2004) The ALARA concept in pediatric CR and DR: dose reduction in pediatric radiographic exams - a white paper conference executive summary. Pediatr Radiol 34(Suppl 3): S162-164

5. Brenner DJ, Hall EJ (2007) Computed tomography - an increasing source of radiation exposure. N Engl J Med 357:2277-2284

6. Hall EJ, Brenner DJ (2008) Cancer risks from diagnostic radiology. Br J Radiol 81:362-378

7. Goske MJ, Applegate KE, Boylan J et al (2008) The 'Image Gently' campaign: increasing CT radiation dose awareness through a national education and awareness program. Pediatr Radiol 38:265-269

8. Strauss KJ, Goske MJ, Frush DP et al (2009) Image Gently vendor summit: working together for better estimates of pediatric radiation dose from CT. AJR 192:1169-1175

9. Thomas KE, Ahmed BA, Shroff P et al (2008) Estimation of cumulative effective doses from diagnostic and interventional radiological examinations in pediatric oncology patients. Pediatr Radiol 38:S339

10. Chawla SC, Federman N, Nagata KT et al (2008) Estimated cumulative radiation dose from PET/CT in pediatric patients with malignancies - a 5-year retrospective review. Pediatr Radiol 38: S339

11. Adamson PC (2009) Imaging in early phase childhood cancer trials. Pediatr Radiol 39(Suppl 1):S38-41

12. Arch ME, Frush DP (2008) Pediatric body MDCT: a 5-year follow-up survey of scanning parameters used by pediatric radiologists. AJR 191:611-617

13. Thomas KE, Wang B (2008) Age-specific effective doses for pediatric MSCT examinations at a large children's hospital using DLP conversion coefficients: a simple estimation method. Pediatr Radiol 38:645-656
14. Kaste SC (2009) Imaging challenges: a US perspective on controlling exposure to ionizing radiation in children with cancer. Pediatr Radiol 39(Suppl 1):S74-79

15. Furth C, Denecke T, Steffen I et al (2006) Correlative imaging strategies implementing CT, MRI, and PET for staging of childhood Hodgkin disease. J Pediatr Hematol Oncol 28:501-512

16. Schenk JP, Graf N, Gunther P et al (2008) Role of MRI in the management of patients with nephroblastoma. Eur Radiol 18:683691

17. Siegel MJ, Jaju A (2008) MR imaging of neuroblastic masses. Magn Reson Imaging Clin N Am 16:499-513 vi

18. Racadio JM (2009) Controlling radiation exposure during interventional procedures in childhood cancer patients. Pediatr Radiol 39(Suppl 1):S71-73

19. Reaman GH (2009) What, why, and when we image: considerations for diagnostic imaging and clinical research in the Children's Oncology Group. Pediatr Radiol 39(Suppl 1):S42-45

20. Shankar LK, Van den Abbeele A, Yap J et al (2009) Considerations for the use of imaging tools for phase II treatment trials in oncology. Clin Cancer Res 15:1891-1897

21. Barnacle AM, McHugh K (2006) Limitations with the response evaluation criteria in solid tumors (RECIST) guidance in disseminated pediatric malignancy. Pediatr Blood Cancer 46:127-134

22. Suzuki C, Jacobsson H, Hatschek T et al (2008) Radiologic measurements of tumor response to treatment: practical approaches and limitations. Radiographics 28:329-344

23. Gallamini A, Hutchings M, Avigdor A et al (2008) Early interim PET scan in Hodgkin lymphoma: where do we stand? Leuk Lymphoma 49:659-662

24. Juweid ME, Stroobants S, Hoekstra OS et al (2007) Use of positron emission tomography for response assessment of lymphoma: consensus of the Imaging Subcommittee of International Harmonization Project in Lymphoma. J Clin Oncol 25:571-578

25. Schoder H, Moskowitz C (2008) PET imaging for response assessment in lymphoma: potential and limitations. Radiol Clin North Am 46:225-241 viii

26. Furth C, Steffen IG, Amthauer H et al (2009) Early and late therapy response assessment with $[18 \mathrm{~F}]$ fluorodeoxyglucose positron emission tomography in pediatric Hodgkin's lymphoma: analysis of a prospective multicenter trial. J Clin Oncol. Aug 10 [Epub ahead of print]

27. Pfluger T, Schmied C, Porn U et al (2003) Integrated imaging using MRI and 123I metaiodobenzylguanidine scintigraphy to improve sensitivity and specificity in the diagnosis of pediatric neuroblastoma. AJR 181:1115-1124

28. Sharp SE, Shulkin BL, Gelfand MJ et al (2009) 123I-MIBG scintigraphy and 18F-FDG PET in neuroblastoma. J Nucl Med 50:1237-1243

29. Kushner BH (2004) Neuroblastoma: a disease requiring a multitude of imaging studies. J Nucl Med 45:1172-1188

30. Meisel JA, Guthrie KA, Breslow NE et al (1999) Significance and management of computed tomography detected pulmonary nodules: a report from the National Wilms Tumor Study Group. Int J Radiat Oncol Biol Phys 44:579-585

31. Owens CM, Veys PA, Pritchard J et al (2002) Role of chest computed tomography at diagnosis in the management of Wilms' tumor: a study by the United Kingdom Children's Cancer Study Group. J Clin Oncol 20:2768-2773

32. Laprise NK, Hanusik R, Fitzgerald TJ et al (2009) Developing a multi-institutional PACS archive and designing processes to manage the shift from a film to a digital-based archive. J Digit Imaging 22:15-24

33. Erberich SG, Silverstein JC, Chervenak A et al (2007) Globus MEDICUS - federation of DICOM medical imaging devices into healthcare grids. Stud Health Technol Inform 126:269-278 